OPEN ACCESS

Edited by:

Hongyu An

Washington University in St. Louis,

United States

Reviewed by:

Nicola Amoroso,

University of Bari Aldo Moro, Italy

Bo Gao,

Affiliated Hospital of Guizhou Medical

University, China

${ }^{*}$ Correspondence:

Ludovica Griffanti

ludovica.griffanti@psych.ox.ac.uk

tThese authors have contributed equally to this work and share first authorship

Specialty section:

This article was submitted to Applied Neuroimaging, a section of the journal Frontiers in Neurology

Received: 04 August 2021 Accepted: 06 October 2021 Published: 29 October 2021

Citation:

Griffanti L, Raman B, Alfaro-Almagro F, Filippini N, Cassar MP, Sheerin F, Okell TW, Kennedy McConnell FA, Chappell MA, Wang C, Arthofer C, Lange FJ, Andersson J, Mackay CE, Tunnicliffe EM, Rowland $M$,

Neubauer S, Miller KL, Jezzard $P$ and Smith SM (2021) Adapting the UK

Biobank Brain Imaging Protocol and Analysis Pipeline for the C-MORE Multi-Organ Study of COVID-19

Survivors. Front. Neurol. 12:753284.

doi: 10.3389/fneur.2021.753284

\section{Adapting the UK Biobank Brain Imaging Protocol and Analysis Pipeline for the C-MORE Multi-Organ Study of COVID-19 Survivors}

Ludovica Griffanti ${ }^{1,2 *}$, Betty Raman ${ }^{3,4 t}$, Fidel Alfaro-Almagro ${ }^{2}$, Nicola Filippini ${ }^{5}$, Mark Philip Cassar ${ }^{3}$, Fintan Sheerin ${ }^{6}$, Thomas W. Okell ${ }^{2}$, Flora A. Kennedy McConnell ${ }^{7,8,9}$, Michael A. Chappell ${ }^{2,7,8,9}$, Chaoyue Wang ${ }^{2}$, Christoph Arthofer ${ }^{2}$, Frederik J. Lange ${ }^{2}$, Jesper Andersson ${ }^{2}$, Clare E. Mackay ${ }^{1,10}$, Elizabeth M. Tunnicliffe ${ }^{3}$, Matthew Rowland ${ }^{11}$, Stefan Neubauer ${ }^{3,4}$, Karla L. Miller ${ }^{2}$, Peter Jezzard ${ }^{2}$ and Stephen M. Smith ${ }^{2}$

${ }^{1}$ Department of Psychiatry, Wellcome Centre for Integrative Neuroimaging, Oxford Centre for Human Brain Activity, University of Oxford, Oxford, United Kingdom, ${ }^{2}$ Nuffield Department of Clinical Neurosciences, Wellcome Centre for Integrative Neuroimaging (WIN FMRIB), University of Oxford, Oxford, United Kingdom, ${ }^{3}$ Division of Cardiovascular Medicine, Radcliffe Department of Medicine, Oxford Biomedical Research Centre (BRC) National Institute for Health Research (NIHR), University of Oxford, Oxford, United Kingdom, ${ }^{4}$ Radcliffe Department of Medicine, British Heart Foundation Centre for Research Excellence, University of Oxford, Oxford, United Kingdom, ${ }^{5}$ Istituto di Ricovero e Cura a Carattere Scientifico (IRCCS) San Camillo Hospital, Venice, Italy, ${ }^{6}$ Department of Radiology, Oxford University Hospitals National Health Service (NHS) Foundation Trust, Oxford, United Kingdom, ${ }^{7}$ Mental Health \& Clinical Neurosciences, School of Medicine, University of Nottingham, Nottingham, United Kingdom, ${ }^{8}$ Sir Peter Mansfield Imaging Centre, School of Medicine, University of Nottingham, Nottingham, United Kingdom, ${ }^{9}$ Nottingham Biomedical Research Centre, Queens Medical Centre, University of Nottingham, Nottingham, United Kingdom, ${ }^{10}$ Department of Psychiatry, University of Oxford, Oxford, United Kingdom,

${ }^{11}$ Nuffield Department of Clinical Neuroscience, University of Oxford, Oxford, United Kingdom

SARS-CoV-2 infection has been shown to damage multiple organs, including the brain. Multiorgan MRI can provide further insight on the repercussions of COVID-19 on organ health but requires a balance between richness and quality of data acquisition and total scan duration. We adapted the UK Biobank brain MRI protocol to produce high-quality images while being suitable as part of a post-COVID-19 multiorgan MRI exam. The analysis pipeline, also adapted from UK Biobank, includes new imaging-derived phenotypes (IDPs) designed to assess the possible effects of COVID-19. A first application of the protocol and pipeline was performed in 51 COVID-19 patients post-hospital discharge and 25 controls participating in the Oxford C-MORE study. The protocol acquires high resolution $T_{1}, T_{2}$-FLAIR, diffusion weighted images, susceptibility weighted images, and arterial spin labelling data in $17 \mathrm{~min}$. The automated imaging pipeline derives 1,575 IDPs, assessing brain anatomy (including olfactory bulb volume and intensity) and tissue perfusion, hyperintensities, diffusivity, and susceptibility. In the C-MORE data, IDPs related to atrophy, small vessel disease and olfactory bulbs were consistent with clinical radiology reports. Our exploratory analysis tentatively revealed some group differences between recovered COVID-19 patients and controls, across 
severity groups, but not across anosmia groups. Follow-up imaging in the C-MORE study is currently ongoing, and this protocol is now being used in other large-scale studies. The protocol, pipeline code and data are openly available and will further contribute to the understanding of the medium to long-term effects of COVID-19.

Keywords: UK Biobank (UKB), brain magnetic resonance imaging (MRI), COVID-19, SARS-CoV-2, multi-organ MRI

\section{INTRODUCTION}

Since its outbreak in 2019, there has been a global effort to understand the impact of SARS-CoV-2 infection on organ health, both in the acute and medium-to-long term phases. Despite being predominantly a respiratory illness, emerging data suggest that damage to multiple organs is common, particularly among those with moderate to severe infections $(1,2)$. A holistic approach to systematically assess the health of multiple vital organs could therefore be advantageous.

Recently we set out to evaluate the medium-term effects of COVID-19 on multiple vital organs (brain, lungs, heart, liver, and kidneys) using magnetic resonance imaging (MRI) in the "Capturing MultiORgan Effects of COVID-19" (CMORE) study (3). A concise yet comprehensive protocol sensitive to the diverse range of COVID-19 manifestations was needed to ensure multiorgan coverage over a reasonable time frame. Incorporation of brain imaging was particularly vital in light of the neuroimaging findings observed in COVID19 patients (4-6) and of the increasing number of studies suggesting a high prevalence of neurological symptoms, mental health abnormalities, and cognitive impairment in survivors (7-10). Reports of subclinical pathology including ischaemic and haemorrhagic events in patients recovering from moderate to severe infections have also raised concerns about the long-term neurological damage caused by COVID-19 (11).

In 2015, the UK Biobank (UKB) imaging study was launched with the primary aim of improving disease prevention, diagnosis, and treatment through insights gained from high-quality imaging (12). As part of this effort, comprehensive imaging of the brain and other organs were planned in 100,000 patients of the original UKB cohort. To date, more than 40,000 patients have been scanned (as of May 2021). To facilitate high throughput image processing of brain images, an automated image analysis pipeline was also developed (13), permitting rapid and reproducible image analysis of large datasets. The pipeline also extracts so-called imaging derived phenotypes (IDPs), quantitative measures that can be easily used and interpreted also by non-imaging experts. In view of this rich resource, the C-MORE study set out to align their postCOVID neuroimaging with the UKB protocol, however, there were two challenges to overcome. First, the standard 30-min UKB brain protocol was too long to be directly incorporated into the 70-min multiorgan protocol. Second, the existing UKB protocol and analysis pipeline was not customised to extract COVID-19 specific imaging markers of interest.
In this study, we sought to describe how we adapted the UKB brain MRI protocol to: (1) achieve an optimal scan time of under $20 \mathrm{~min}$ for incorporation into the multiorgan protocol; (2) augment its sensitivity to COVID-19 specific pathology (3) exploit its technical advances and enable future data comparison and merging. As an extension to the original study (3), the pipeline has been expanded to generate 1,467 additional IDPs (cortical grey matter density measures, cortical thickness and area from FreeSurfer, olfactory bulb volume and intensity, mean diffusivity within the tracts' normal appearing white matter, and perfusion metrics in the white matter, for a total of 1,575 IDPs) and includes an improved estimate of quantitative susceptibility mapping of subcortical structures. We then assessed the agreement between IDPs and radiology reports. Regarding the exploratory analyses on the impact of COVID-19 on the brain in the C-MORE dataset, we expanded the group comparison between patients and controls to include additional new IDPs, and investigated group differences based on disease severity and the presence or absence of anosmia.

\section{METHODS}

\section{MRI Acquisition Protocol}

Table 1 reports the details of the brain MRI sequences included in the multiorgan COVID-19 protocol, in comparison with the UKB protocol. Below we describe the rationale for the inclusion of these MRI sequences and, where applicable, deviations from the UKB protocol.

The scanner used in UKB is a standard Siemens Skyra 3T running VD13A, with a standard Siemens 32-channel RF receive head coil. The multiorgan COVID-19 protocol was setup on a Siemens Prisma 3T running VE11C, with a Siemens 20-channel head coil.

\section{Rationale for Using Specific Brain MRI Sequences in the COVID-19 Multiorgan Protocol}

\section{High-Resolution $\mathrm{T}_{1}$-Weighted Image}

This structural sequence is primarily used to study grey matter (GM) macroscopic anatomy in both cortical and subcortical brain regions. By exploiting differences in the interaction of water with surrounding tissues (tissue $\mathrm{T}_{1}$ relaxation times), this sequence provides strong contrast between grey and white matter. GM reductions have been widely associated with Alzheimer's disease and age-related cognitive dysfunction (14). Emerging evidence shows that COVID-19 may exacerbate or even cause cognitive problems $(8,15,16)$, potentially contributing to a new wave of dementia and multi-morbidity 
TABLE 1 | Brain MRI sequences of the COVID-19 multiorgan protocol: acquisition details and comparison with UKB protocol.

\begin{tabular}{|c|c|c|c|c|c|}
\hline Modality & $\begin{array}{l}\text { Acquisition } \\
\text { time }\end{array}$ & Resolution (mm) & Matrix & Key parameters & Biobank match \\
\hline $\mathrm{T}_{1}$ (MPRAGE) & $4: 54$ & $1.0 \times 1.0 \times 1.0$ & $256 \times 256 \times 208$ & $\mathrm{TI} / \mathrm{TR}=800 / 2,000 \mathrm{~ms}, \mathrm{R}=2$ & Exact \\
\hline $\mathrm{T}_{2}$ FLAIR (SPACE) & $4: 32$ & $1.0 \times 1.0 \times 1.05$ & $256 \times 256 \times 192$ & $\mathrm{TI} / \mathrm{TR} / \mathrm{TE}=\mathbf{1 , 8 0 0 / 5 , 0 0 0 / 3 8 6} \mathrm{ms}, \mathrm{R}=3$ & Very similar \\
\hline Diffusion MRI & $1: 33$ & $2.0 \times 2.0 \times 2.0$ & $104 \times 104 \times 72$ & $\begin{array}{l}\mathrm{TR}=8,500 \mathrm{~ms}, 3 \text { dirs, } \mathrm{b}=0,1,000 \\
\mathrm{~s} / \mathrm{mm}^{2}, \text { blip-reversed } \mathrm{b}=0\end{array}$ & $\begin{array}{l}\text { Subset: 3-scan trace } \\
\text { only }\end{array}$ \\
\hline $\begin{array}{l}\text { Susceptibility-weighted } \\
\text { imaging }\end{array}$ & $2: 08$ & $0.9 \times 0.9 \times 3.0$ & $256 \times 232 \times 48$ & TE1/TE2/TR $=9.4 / 20 / 27 \mathrm{~ms}, \mathrm{R}=2$ & Slightly lower resolution \\
\hline $\begin{array}{l}\text { ASL } \\
\text { (multi-slice multi-PLD PCASL) }\end{array}$ & $3: 41$ & $3.4 \times 3.4 \times 4.5$ & $64 \times 64 \times 24$ & $\begin{array}{l}\text { Variable TR to minimise deadtime (max. } \\
4,500 \mathrm{~ms} \text { ), label duration }=1,400 \mathrm{~ms} \text {, six } \\
\text { PLDs }=300: 300: 1,800 \mathrm{~ms}, 5 \text { reps of all } \\
\text { PLDs, } 1 \text { M0 calibration image }\end{array}$ & $\begin{array}{l}\text { A similar ASL protocol } \\
\text { has recently added to } \\
\text { UKB for } \\
\text { post-COVID-19 } \\
\text { scanning }\end{array}$ \\
\hline Total scanning time & $16: 48$ & & & & \\
\hline \multicolumn{6}{|l|}{ UK Biobank } \\
\hline $\mathrm{T}_{1}$ (MPRAGE) & $4: 54$ & $1.0 \times 1.0 \times 1.0$ & $256 \times 256 \times 208$ & $\mathrm{TI} / \mathrm{TR}=800 / 2,000 \mathrm{~ms}, \mathrm{R}=2$ & \\
\hline $\mathrm{T}_{2}$ FLAIR (SPACE) & $5: 52$ & $1.0 \times 1.0 \times 1.05$ & $256 \times 256 \times 192$ & $\mathrm{Tl} / \mathrm{TR} / \mathrm{TE}=\mathbf{1 , 8 0 0 / 5 , 0 0 0 / 3 9 5} \mathbf{m s}, \mathrm{R}=2$ & \\
\hline Diffusion MRI & $7: 08$ & $2.0 \times 2.0 \times 2.0$ & $104 \times 104 \times 72$ & $\begin{array}{l}\mathrm{TR}=3,600 \mathrm{~ms}, 50 \text { dirs/shell, } \mathrm{b}=0 \\
\text { 1,000, } 2,000 \mathrm{~s} / \mathrm{mm}^{2}, \mathrm{MB}=3 \\
\text { blip-reversed } \mathrm{b}=0\end{array}$ & \\
\hline $\begin{array}{l}\text { Susceptibility-weighted } \\
\text { imaging }\end{array}$ & $2: 34$ & $0.8 \times 0.8 \times 3.0$ & $288 \times 256 \times 48$ & $\mathrm{TE} 1 / \mathrm{TE2} / \mathrm{TR}=9.4 / 20 / 27 \mathrm{~ms}, \mathrm{R}=2$ & \\
\hline Task fMRI & $4: 13$ & $2.4 \times 2.4 \times 2.4$ & $88 \times 88 \times 64$ & $\mathrm{TE} / \mathrm{TR}=39 / 735 \mathrm{~ms}, \alpha=52^{\circ}, \mathrm{MB}=8$ & \\
\hline Resting fMRI & $6: 10$ & $2.4 \times 2.4 \times 2.4$ & $88 \times 88 \times 64$ & $\mathrm{TE} / \mathrm{TR}=39 / 735 \mathrm{~ms}, \alpha=52^{\circ}, \mathrm{MB}=8$ & \\
\hline Total scanning time & $30: 51$ & & & & \\
\hline
\end{tabular}

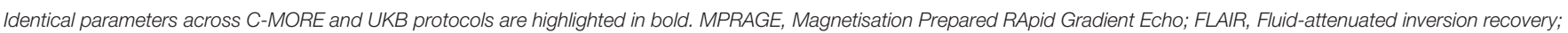

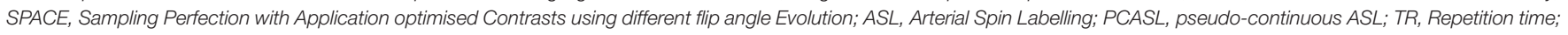

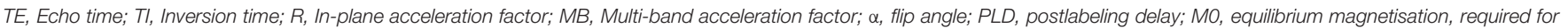

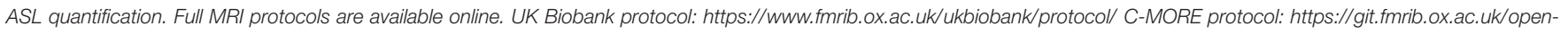
science/analysis/c-more-brain-mri.

in the future (11). A recent study (6), however, found GM increases in recovered hospitalised COVID-19 patients (MRI about 3 months from onset), relative to controls. High-resolution $\mathrm{T}_{1}$ is also critical for surface generation and calculations of cross-subject and cross-modality alignments, needed in order to process all other brain modalities and to perform voxel-wise analyses. This protocol was exactly matched to the one used in UKB.

\section{$\mathrm{T}_{2}$-Weighted Fluid Attenuated Inversion Recovery ( $\mathrm{T}_{2}$-FLAIR)}

This structural sequence is commonly used in clinical practice, for example to characterise white matter (WM) hyperintensities (WMH). In $\mathrm{T}_{2}$-weighted images, the contrast is dominated by signal decay from interactions between water molecules $\left(\mathrm{T}_{2}\right.$ relaxation times) and image intensity is related to alterations to tissue compartments typically associated with pathology. While a recent review (17) described WMHs as the most frequently reported brain abnormalities in adults with COVID19 in the acute and subacute phases, other patterns of $\mathrm{T}_{2}$ FLAIR alterations have also been reported, including signal abnormalities in the medial temporal lobe and non-confluent multifocal WM hyperintense lesions with variable enhancement associated with haemorrhagic lesions (15). Loss of smell, a characteristic symptom of COVID-19, has been linked with abnormal olfactory bulb $\mathrm{T}_{2}$-FLAIR signal (18) and atrophy (19), although these findings remain controversial (20, 21). Given that anosmia is also a common feature of Parkinsonian disease, in which chronic neuroinflammation is thought to play a role, it has been suggested that patients could be at risk of parkinsonism following infection with COVID-19 (22). Therefore, it remains important to monitor the olfactory system in COVID-19 survivors given the potential for long-term postviral Parkinsonism $(22,23)$. With respect to the UKB protocol, the sequence has been shortened by $80 \mathrm{~s}$, mainly by increasing the in-plane acceleration factor from $\mathrm{R}=2$ to $\mathrm{R}=3$, keeping the high spatial resolution unchanged.

\section{Diffusion-Weighted MR Imaging (dMRI)}

This sequence measures the ability of water molecules to move within their local environment. The UKB dMRI sequence includes 100 diffusion-encoding directions across 2 b-shells, enabling measurement of the random motion of water molecules to infer information about WM microstructural properties and delineate the gross axonal organisation of the brain. In this multiorgan protocol, we acquired just 3 orthogonal diffusion directions, as commonly done in clinical practice. This allowed us to substantially decrease the acquisition time (from 7:08 to 1:33), while still being able to estimate mean diffusivity (MD), important for example in the assessment 
of ischaemic injury. Cases of brain ischaemic injury, acute disseminated encephalomyelitis, and encephalitis have been reported in COVID-19 cases (7), while Lu et al., reported significant differences in dMRI-derived measures, including MD, in COVID-19 with respect to controls (6).

\section{Susceptibility-Weighted MR Imaging (swMRI)}

This sequence is sensitive to magnetised tissue constituents, including iron, calcium, and iron, based on their shifted magnetic susceptibility compared to water (24). Here, we process the magnitude images to calculate $\mathrm{T}_{2}^{*}$ maps (which reflect compartmentalisation of these constituents) and quantitative susceptibility maps (QSM, which reflect the mean magnetic susceptibility) (25). SwMRI is commonly used in clinical settings to study diverse pathologic conditions, such as traumatic brain injury, stroke, and haemorrhages. It can also be used to define vascular malformations, brain tumours, cerebral microbleeds, intracranial calcifications, and iron deposits. Haemorrhagic lesions and extensive and isolated WM microhemorrhages have frequently been reported in $\operatorname{COVID}-19$ (7, 15, 17, 26). The highest iron concentration in the adult brain is found in the basal ganglia and is known to increase with age (27). However, focal accumulation of iron is associated with neurodegenerative disorders and has been linked to inflammation (28). The only change in this sequence with respect to the one used in the UKB protocol is a small reduction in in-plane spatial resolution (from $0.8 \times 0.8 \mathrm{~mm}$ iso to $0.9 \times 0.9$ iso), shortening the acquisition time by $\sim 30 \mathrm{~s}$.

\section{Arterial Spin Labelling (ASL)}

Brain perfusion has not previously been assessed in UKB, although the recently-initiated UKB post-COVID-19 imaging study has for the first time included a fast, modest ASL protocol. For C-MORE we included a similar multi-slice multipost label delay (multi-PLD) pseudo-continuous ASL (PCASL) sequence with pre-saturation and two global inversion pulses for background suppression, but here using a $1.4 \mathrm{~s}$ labelling duration and a with multi-slice 2D EPI readout (29) with $45.2 \mathrm{~ms}$ to acquire each slice in ascending order. This sequence is used to quantify tissue perfusion, i.e., cerebral blood flow (CBF), and Arterial Transit Time (ATT) by using the water in arterial blood as an endogenous contrast agent. Given the evidence of vascular damage (haemorrhages, strokes, microbleeds, vascular lesions) in some COVID-19 patients (7) in the acute setting, we deemed it to be particularly important to assess brain perfusion in this protocol.

\section{Dataset: the C-MORE Study}

This protocol was first used in the C-MORE study. Details of the study are described elsewhere (3). Briefly, we included 58 patients with moderate to severe acute respiratory distress syndrome Coronavirus-2 (SARS-CoV-2 infection). All patients tested positive on a reverse transcription and polymerase chain reaction (RT-PCR) nasopharyngeal swab test and required hospital admission for more than $48 \mathrm{~h}$ between 14th March and 25th May 2020. The severity of disease during hospital admission was graded as per the WHO ordinal scale for clinical improvement.

Thirty controls group-matched for age, sex, body mass index (BMI), and risk factors (smoking, diabetes, and hypertension) were included in the study. Controls were non-hospitalised subjects (invited from the community) without symptoms or signs of a respiratory tract infection of coronavirus disease, who were screened for SARS-CoV-2 antibodies and tested negative. Subjects with contraindications to magnetic resonance imaging (metal implant in body, known claustrophobia, pacemakers, contrast allergy) and severe comorbidities-endstage renal, cardiac, liver, neurological disease-were excluded from the study.

The multiorgan MRI scan was carried out 2-3 months from disease onset (median 2.3, IQR 2.06-2.53 months), imaging the brain, lungs, heart, liver, and kidneys (3T Prisma, Siemens Healthineers, Erlangen, Germany). The total acquisition time was $70 \mathrm{~min}$.

Details on clinical symptoms or signs, vitals and laboratory findings during admission were extracted from electronic medical records, while additional evaluations were performed at the time of scan [see (3) for details]. The severity of disease during hospital admission was graded as per the WHO ordinal scale for clinical improvement. This study was registered at ClinicalTrials.gov (NCT04510025) and approved in the United Kingdom by the North West Preston Research Ethics Committee (reference 20/NW/0235).

\section{Analysis Pipeline}

In this section, we describe the IDPs derived from the automated pipeline. When applicable, we detail pipeline modifications with respect to $\mathrm{UKB}$. The main $\mathrm{UKB}$ pipeline is openly available (https://www.fmrib.ox.ac.uk/ukbiobank/) and new IDPs will be included in the next version. Modified or additional scripts and support data for the analyses performed in this study are available at (https://git.fmrib.ox.ac.uk/open-science/analysis/cmore-brain-mri). Examples of pipeline outputs for each modality are shown in Figure 1.

Image processing was largely based around tools from FSL (FMRIB Software Library) (30). Data were corrected for gradient and EPI distortions and aligned with each other using linear alignment (31) (between modalities, within-subject). Subjects were then aligned into standard template space (MNI 152) using FNIRT non-linear alignment driven by $\mathrm{T}_{1}$-weighted images (32) and aligned to a study-specific template using MMORF non-linear alignment driven by both the $\mathrm{T}_{1}$ and $\mathrm{T}_{2}$-FLAIR images (33).

\section{$\mathrm{T}_{1}$ and $\mathrm{T}_{\mathbf{2}}$-FLAIR}

As part of the UKB pipeline, several global, and regional volume measures are extracted from the $\mathrm{T}_{1}$ scans with SIENAX (34) (11 IDPs, including head size). $\mathrm{T}_{1}$ images are segmented probabilistically into different tissue types with FAST (35), and from the grey matter partial volume estimates (PVE) (Figure 1A) the average volume (GM density) is calculated within 139 ROIs (defined by the combination of parcellations from several atlases: Harvard-Oxford cortical and subcortical atlases, and 

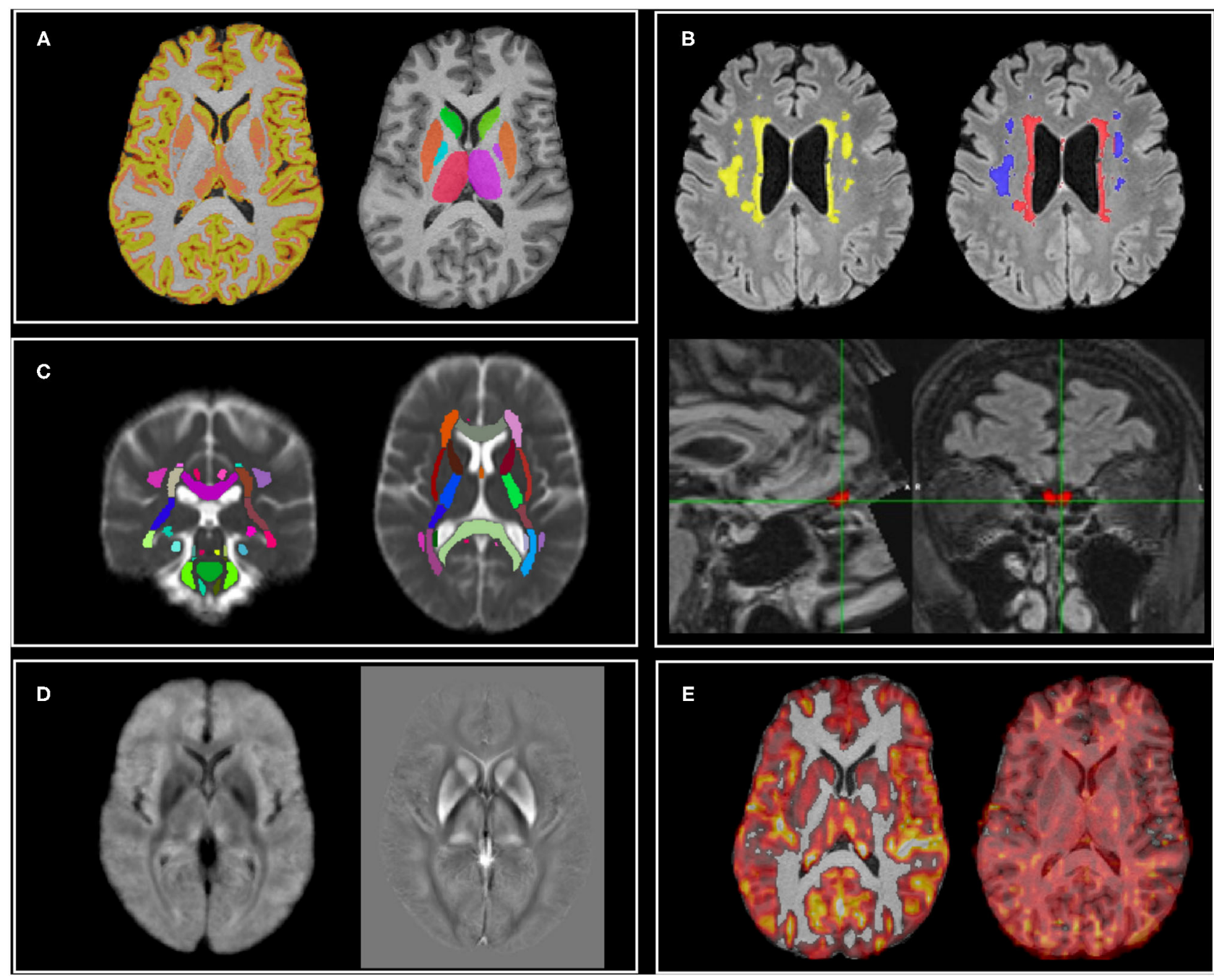

FIGURE 1 | MRI modalities and examples of outputs of the analysis pipeline. (A) T1: cortical volumes (left-GM partial volume estimate shown as example) and subcortical volumes (right); (B) T2-FLAIR: white matter hyperintensities (total in yellow, periventricular in red, deep in blue) and olfactory bulb volume and intensity; (C) dMRI: mean diffusivity of major white matter tracts (average MD image across subjects shown for display purposes); (D) Susceptibility weighted imaging (average images across subjects shown for display purposes): $T_{2}^{\star}$ (left) and QSM (right); (E) ASL: non-partial volume corrected cerebral blood flow (left-lower threshold set to $20 \mathrm{ml} / 100 \mathrm{~g} / \mathrm{min}$ for display purposes) and arterial transit time (right).

Diedrichsen cerebellar atlas, see Supplementary Table 1). The volume of 15 subcortical structures is extracted using FIRST (36) (Figure 1A).

The $\mathrm{T}_{1}$ images are also processed with FreeSurfer using $\mathrm{T}_{2}$-FLAIR in conjunction with $\mathrm{T}_{1}$ for modelling the cortical surface $(37,38)$. Derived IDPs include subcortical segmentation metrics $(39,40)$, regional surface area, volume, and mean cortical thickness from different parcellations [Desikan-Killiany, Brodmann, Desikan-Killiany-Tourville DKT (41), Destrieux], and grey-white intensity contrasts (expressed as the fractional contrast between white and grey matter intensities as sampled either side of the grey-white cortical boundary) (42) (1,273 IDPs).

The total volume of WMHs was calculated with BIANCA (43) using both $\mathrm{T}_{1}$ and $\mathrm{T}_{2}$-FLAIR images and the UK Biobank training file (available from the UKB pipeline). WMH masks were visually inspected and manually edited if needed, blind to diagnosis (Figure 1B).

In addition to the UKB IDPs, periventricular WMH (PWMH) and deep WMH (DWMH) volumes, defined as being less or more than $10 \mathrm{~mm}$ distant from the lateral ventricles, respectively $(44,45)$ (Figure 1B), were extracted as subsets of WMHs (3 WMH IDPs in total).

$\mathrm{T}_{1}$ and $\mathrm{T}_{2}$-FLAIR images were also used to extract two IDPs to assess potential abnormalities in the olfactory bulb (OB) (Figure 1B). This was achieved in three steps. In the first step, a multimodal, non-linear template was constructed from $\mathrm{T}_{1}$ and $\mathrm{T}_{2}$-FLAIR images of $25 \mathrm{UKB}$ participants with a mean age of $63 \pm 8$ years and 15/25 (60\%) men. Average intensity and shape templates were generated with an iterative, multi-resolution approach $(46,47)$, by minimising both the 
intensity difference between the template and each subject and the deformation required to warp the template to each subject. The first iteration was initialised with unbiased, affine $T_{1}$ and $\mathrm{T}_{2}$-FLAIR templates which were constructed from the same set of subjects and rigidly aligned to the 6th generation nonlinear MNI 152 template (48). Rigid and affine registrations used to align $T_{1}$ and $T_{2}$-FLAIR images within subjects, and $\mathrm{T}_{1}$ images between subjects, respectively, were performed with FLIRT (31). Non-linear registrations between each subject and the template were performed with MultiModal Registration Framework (MMORF) (33), which allowed the simultaneous registration of both modalities, and brain and non-brain tissue. In the second step, the average templates served as a reference space for normalisation and the enhanced SNR enabled clear visualisation of olfactory bulbs (OB). The left and right $\mathrm{OB}$ were manually segmented on the template to create two labels (checked by a neuroanatomy expert). MMORF was used to estimate a deformation field between each individual C-MORE subject's $\mathrm{T}_{1}$ and $\mathrm{T}_{2}$-FLAIR images and the template. Finally, each individual's inverse transformation was used to transform the OB labels from template space back to each individual's native space, where the $\mathrm{OB}$ volume and $\mathrm{OB}$ mean $\mathrm{T}_{2}$-FLAIR intensity (normalised with respect to the median $\mathrm{T}_{2}$-FLAIR intensity in the white matter) measurements were performed (2 IDPs).

\section{Diffusion-Weighted MR Imaging}

Diffusion data were pre-processed with eddy_correct and topup to remove effects of eddy currents, head motion, and susceptibility-induced distortions $(49,50)$, and then processed to generate a mean diffusivity (MD) map. The UKB TBSS pipeline was adapted to take into account the availability of MD only. $\mathrm{MD}$ images were registered to $\mathrm{MNI}$ space combining a linear transformation from the $\mathrm{b}=0$ image to $\mathrm{T}_{1}$ with the non-linear registration from $\mathrm{T}_{1}$ to $\mathrm{MNI}$. Average $\mathrm{MD}$ was then calculated within an average skeleton in standard space for each tract of the JHU atlas (Figure 1C) (48 IDPs). In addition, the analysis was repeated to assess MD in the normal-appearing WM (NAWM), i.e., after excluding voxels where WMHs were present. To achieve this, the linear transformation between $\mathrm{T}_{2}$-FLAIR and the $\mathrm{b}=0$ image was calculated and applied to WMH maps. WMHs were then masked out from the MD map before calculating the mean values in the skeleton's tracts (48 IDPs).

\section{Susceptibility-Weighted MR Imaging}

Both magnitude and phase data acquired from swMRI acquisitions (Figure 1D) were processed to provide quantitative measures reflecting clinically-relevant tissue susceptibility properties. First, as part of the UKB pipeline, magnitude data from two echoes were processed to provide a quantitative mapping of $\mathrm{T}_{2}^{*}$ signal decay times. Median $\mathrm{T}_{2}^{*}$ was calculated within each of 14 major subcortical GM structures (previously derived from the $\mathrm{T}_{1}$ using FIRST) as IDPs. Second, phase data were processed for QSM following a pipeline being developed for UKB (51). Briefly, phase images from each channel were combined using the MCPC-3D-S approach (52), the channelcombined phase images from two echoes were unwrapped using a Laplacian-based algorithm (53) and subsequently combined into one phase image via a weighted-average (54). This phase image was then filtered using the V-SHARP algorithm (55) to remove background field, and susceptibility maps were generated using the iLSQR algorithm (56). Voxels within the ventricles were extracted using a ventricle mask derived from the $\mathrm{T}_{2}$-FLAIR data (using make_bianca_mask) and subsequently, a mixture modelling algorithm (including one Gaussian and two inverse-Gamma models) (57) was applied to the extracted voxels. Susceptibility value of CSF was calculated as the mean value of the main Gaussian distribution and was used as the reference for susceptibility maps. Median susceptibility (CSF-referenced) was calculated in the same 14 subcortical structures as the $\mathrm{T}_{2}^{*}$ IDPs.

\section{Arterial Spin Labelling}

The ASL data were processed using the BASIL ASL tools in FSL to estimate CBF and ATT (Figure 1E). BASIL analysis involved motion correction, distortion correction (using the field map derived from the blip-up/down diffusion data), brain masking, label-control subtraction, and kinetic model fitting, including modelling of the macrovascular component $(58,59)$ and partial volume correction (PVEc) (60). Absolute CBF quantification was achieved via voxel-wise calibration using the first volume of the ASL data, which was acquired with no ASL preparation or background suppression (the M0 calibration volume). The resulting voxel-wise perfusion images were linearly aligned to the $\mathrm{T}_{1}$ structural image using FLIRT with the BBR cost function. Grey matter and white matter PVE maps from FAST were transformed into native ASL space using applywarp with super sampling to integrate the tissue partial volume contributions across the larger ASL native space voxel volumes. A grey matter mask was defined by applying a partial volume threshold of $50 \%$ (i.e., including only voxels of the PVE GM map were the proportion of GM was above 50\%) in the native ASL space; this mask was then used to estimate mean grey matter CBF and ATT from both the non-PVEc and PVEc results (4 IDPs). Similarly, a white matter mask was defined using a partial volume threshold of $80 \%$ (i.e., including only voxels of the PVE WM map were the proportion of WM was above $80 \%$ ); this mask was then used to estimate mean white matter CBF and ATT (4 IDPs).

In summary, the current version of this pipeline allows extraction of 1,575 IDPs (see complete list in Supplementary Table 1).

\section{Quality Control and Neuroradiology Reports}

Qualitative assessment of all brain MRI images from the CMORE dataset was undertaken by an expert neuroradiologist (FS), who commented on the presence of white matter hyperintensities, brain atrophy, ischaemic or haemorrhagic abnormalities, and olfactory bulb size and signal.

All data and main outputs of the analysis pipeline were also manually quality-control checked using the same criteria as the UKB imaging study [details in (13)] and an additional set of visual checks for ASL data ${ }^{1}$

\footnotetext{
${ }^{1}$ https://git.fmrib.ox.ac.uk/open-science/analysis/c-more-brain-mri/-/tree/ master/ASL_pipeline
} 


\section{Exploratory Analyses on the C-MORE Dataset}

As an initial step, we examined the level of agreement between outputs from our automated pipeline against the visual scores provided in the radiology reports (independently from diagnosis). For small vessel disease we compared total WMH volume against visual ratings (none/mild/moderate) using the Kruskal-Wallis test. For brain atrophy we compared total brain volume, total GM and cortical GM (all normalised for head size) between scans classified as normal for age and those reported as showing generalised atrophy using the Mann-Whitney test. Finally, we looked at the volume and intensity on $\mathrm{T}_{2}$-FLAIR in the olfactory bulbs.

Group comparisons of IDPs were then undertaken after Gaussianisation (quantile normalisation) of all continuous variables and after regressing out the following confounds: age, sex, BMI, diastolic and systolic blood pressure, smoking and head size. For the FreeSurfer IDPs, the comparisons were based on the Desikan-Killiany-Tourville DKT (41) atlas (186 IDPs). Unless otherwise stated, comparisons were therefore run on 487 IDPs. The conventional level of statistical significance of $5 \%$ was used without correction for multiple comparisons. Statistical analyses were performed using SPSS Version 27.0 (IBM, Armonk, NY, USA).

We first explored differences in the IDPs between recovered COVID-19 patients and controls (two sample independent $t$-test). We then defined as critical those patients who received organ support (any of the following: positive airway pressure ventilation, intubation, dialysis, vasopressor support) and explored differences across severity groups with one-way ANOVA, 3 groups: critical, non-critical, controls (HC).

To look at potential differences linked to the loss of smell, we performed a group comparison (ANOVA 3 groups: patients with anosmia, patients without anosmia, controls) on the following 26 IDPs: OB volume and intensity (2 IDPs), GM density of the frontal orbital, frontal medial cortex, frontal pole (6 IDPs), volume, area, and cortical thickness of the lateral and medial orbitofrontal cortex (12 IDPs), amygdala and brainstem volume (from FIRST and GM parcellation, 6 IDPs).

Follow-up Pearson's correlations with inflammatory markers (from the blood assay) were performed within the patient group to aid the interpretation of significant group differences.

\section{RESULTS}

Among the 58 patients included in the study, 21 (36\%) required intensive care unit admission and 20/21 required mechanical ventilation (non-invasive ventilation or intubation). Four patients required renal replacement therapy or inotropic support. Steroids were used in $27.6 \%$ of patients. Median duration of hospitalisation was 8.5 days (IQR 5.0-17.0). Patients were assessed between 2 and 3 months from disease-onset at median interval of 2.3 months (IQR 2.06-2.53) and median 1.6 months from discharge (IQR 1.4-1.8). More details on patients' characteristics and admission details are provided in (3). Eighty participants completed the brain sequences of the multiorgan scan. After quality control we excluded four participants (2 for excessive motion on $\mathrm{T}_{1}, 1$ for presence of an extensive lesion compromising registrations, 1 for pipeline failure at brain extraction level) obtaining usable brain imaging data from 51 patients and 25 controls.

Table 2 summarises the main demographic and disease information from the final 76 participants (95\% of the initial sample), together with the number of usable scans per group for each sequence (modality). We also detail the number of radiology reports where a specific comment was made with respect to the amount of small vessel disease, brain atrophy, and olfactory bulbs' characteristics.

The groups were well-matched in terms of demographics and risk factors and showed no significant difference in the patterns of radiological signs. The COVID-19 patients had mildly worse vital signs (higher heart rate and respiratory rate, lower oxygen saturation, and higher body temperature) than controls, although still within the normal range.

\section{Comparison Between IDPs and Neuroradiology Reports}

Figure 2 shows the comparison between IDP values for $\mathrm{WMH}$, total GM volume and $\mathrm{OB}$ volume and intensity and the classifications from the radiology reports (colour-coded according to diagnosis group). For one participant there was no explicit comment on small vessel disease or atrophy. Most of the scans (78\%) were classified as normal. Significant differences in IDPs were observed from the Kruskal-Wallis and MannWhitney tests across small vessel disease (SVD) classes and between atrophy classes, respectively (Supplementary Table 2), suggesting that IDPs are in line with radiology reports. However, this is only a first validation, which needs to take into account the low number of subjects especially in the "moderate SVD" and "generalised atrophy" classes.

Regarding the olfactory bulb, for four participants there was no explicit comment on the size or intensity, or the $\mathrm{OB}$ was rated as "difficult to resolve." The remaining scans were rated as normal in size and signal, with one scan classified as borderline small, but with normal signal, in line with the IDPs relative to OB volume (lower than all scans rated as normal) and $\mathrm{T}_{2}$-FLAIR intensity (value within the 20th percentile of the scans rated as normal) (Figure 2, bottom row).

\section{Results of the Cohort Comparisons Based on Disease Group}

The Supplementary Material contains an overview of the results of the group comparisons between previously hospitalised COVID-19 patients and controls and across severity groups: patients who required organ support (critical), patients who did not require organ support (non-critical), controls (HC). From these exploratory analyses we observed some significant group differences $\left[p_{\text {(uncorr })}<0.05\right]$ and representative examples are shown in Figure 3.

\section{$T_{1}$ and $T_{2}$-FLAIR}

The main differences in the grey matter between COVID19 patients and controls were observed in the frontal lobe. 
TABLE 2 | Demographics, vital signs, and blood test at follow-up (3 months visit) disease details, usable brain MRI scans, and radiology reports details of patients and control participants in the C-MORE study.

$\begin{array}{lcc}\text { Controls } & \text { COVID-19 } & p \text {-value }^{*} \\ (N=25) & (N=51) & \end{array}$

\begin{tabular}{|c|c|c|c|}
\hline \multicolumn{4}{|c|}{ Demographics, cognition, and risk factors } \\
\hline Age (years) & $52.4 \pm 12.8$ & $54.8 \pm 13.4$ & 0.46 \\
\hline $\operatorname{Sex}(M / F)$ & $15 / 10$ & $29 / 22$ & $1^{\#}$ \\
\hline BMI $\left(\mathrm{kg} / \mathrm{m}^{2}\right)$ & $28.8 \pm 7.1$ & $31.1 \pm 6.1$ & 0.15 \\
\hline MoCA & $27.7 \pm 1.9$ & $26.7 \pm 3.2$ & 0.16 \\
\hline $\begin{array}{l}\text { Smoking } \\
\text { (current or ex-smoker/non-smoker) }\end{array}$ & $6 / 19$ & $18 / 33$ & $0.43^{\#}$ \\
\hline Hypertension (yes/no) & $5 / 20$ & $18 / 33$ & $0.19^{\#}$ \\
\hline Diabetes (yes/no) & $2 / 23$ & $8 / 43$ & $0.48^{\#}$ \\
\hline \multicolumn{4}{|l|}{ Disease details } \\
\hline Required organ support (yes/no) & - & $17 / 34$ & \\
\hline Anosmia (yes/no) & - & $24 / 27$ & \\
\hline \multicolumn{4}{|l|}{ Vital signs at 3 months follow-up visit } \\
\hline Systolic blood pressure (mmHg) & $135.0 \pm 17.2$ & $137.9 \pm 15.9$ & 0.46 \\
\hline Diastolic blood pressure $(\mathrm{mmHg})$ & $75.6 \pm 15.2$ & $78.8 \pm 10.3$ & 0.33 \\
\hline Heart rate (bpm) & $69.5 \pm 12.8$ & $76.3 \pm 13.7$ & 0.044 \\
\hline Respiratory rate (breaths per minute) & $16.0 \pm 2.5$ & $17.8 \pm 3.0$ & 0.012 \\
\hline Oxygen saturation (\%) & $97.2 \pm 1.5$ & $96.1 \pm 1.5$ & 0.005 \\
\hline Temperature $\left({ }^{\circ} \mathrm{C}\right)$ & $36.5 \pm 0.16$ & $36.6 \pm 0.18$ & 0.033 \\
\hline $\begin{array}{l}\text { Blood tests at } 3 \text { months follow-up } \\
\text { visit }\end{array}$ & & $(N=50)$ & \\
\hline White cell count, $\times 10^{9} / \mathrm{L}$ & $6.5 \pm 1.7$ & $6.5 \pm 1.8$ & 0.85 \\
\hline Neutrophil count, $\times 10^{9} / \mathrm{L}$ & $3.8 \pm 1.4$ & $3.7 \pm 1.4$ & 0.8 \\
\hline Lymphocyte count, $\times 10^{9} / \mathrm{L}$ & $2.0 \pm 0.5$ & $1.9 \pm 0.5$ & 0.76 \\
\hline Monocyte count, $\times 10^{9} / \mathrm{L}$ & $0.5 \pm 0.1$ & $0.6 \pm 0.2$ & 0.47 \\
\hline \multicolumn{4}{|l|}{ Usable scans } \\
\hline $\mathrm{T}_{1}$ & 25 & 51 & \\
\hline $\mathrm{T}_{2}$-FLAIR & 25 & 50 & \\
\hline dMRI & 25 & 51 & \\
\hline swMRI & 23 & 51 & \\
\hline ASL & 25 & 49 & \\
\hline \multicolumn{4}{|l|}{ Radiology reports } \\
\hline $\begin{array}{l}\text { Small vessel disease } \\
\text { (none/mild/moderate) }\end{array}$ & $19 / 4 / 1$ & $38 / 11 / 1$ & $0.77^{\#}$ \\
\hline $\begin{array}{l}\text { Atrophy (normal for age/generalised } \\
\text { atrophy) }\end{array}$ & $21 / 3$ & $49 / 1$ & $0.09^{\#}$ \\
\hline $\begin{array}{l}\text { Abnormal olfactory bulb } \\
\text { (normal/borderline small/N/A) }\end{array}$ & 24/0/1 & $47 / 1 / 3$ & $1^{\#}$ \\
\hline
\end{tabular}

BMI, Body Mass Index; MoCA, Montreal Cognitive Assessment; bpm, beats per minute; FLAIR, Fluid Attenuated Inversion Recovery; dMRI, diffusion-weighted MR imaging; swMRI, susceptibility-weighted MR imaging; ASL, arterial spin labelling; N/A, information not reported (for olfactory bulb this includes cases classified as difficult to resolve). * Independent $t$-test (unless otherwise stated). "Fisher's Exact Test or Pearson's ChiSquare. Significant differences are highlighted in bold.

COVID-19 patients had lower GM density in the left superior frontal gyrus (Figure 3A) and a lower volume and mean cortical thickness in the caudal portion of the left middle frontal gyrus. The GM density and volume of the left superior frontal gyrus, the GM density of the right inferior frontal gyrus (pars triangularis), the cortical thickness of the left lateral orbitofrontal gyrus and the cortical thickness of the left pars orbitalis of the inferior frontal gyrus were significantly different across severity groups, with lowest GM volume in the patients who received organ support (Figure 3B) (details in Supplementary Material). Within the COVID-19 patients, the GM density of the left superior frontal gyrus was negatively correlated with systemic markers of inflammation (white cell count: $r=-0.34, p=$ 0.015 ; neutrophils count: $r=-0.28, p=0.049$; lymphocytes count: $r=-0.33, p=0.019$; monocytes count: $r=-0.31, p$ $=0.03$ ) (Figure 3C) and the cortical thickness of the left lateral orbitofrontal cortex was negatively correlated with lymphocytes count $(r=-0.32, p=0.02)$.

In addition, COVID-19 patients had decreased GM metrics (GM density, volume, thickness, or area) with respect to controls in the following areas: left hippocampus (lower volume), left superior division of the lateral occipital cortex (lower GM density), right middle temporal gyrus (lower thickness, lowest in the non-critical group), right superior temporal gyrus (lower thickness), right inferior parietal (lower thickness), right supramarginal gyrus (lower thickness, lowest in the non-critical group), right isthmus of the cingulate gyrus (lower area and volume, lowest in the non-critical group), left isthmus of the cingulate gyrus (lower volume), right cuneus (lower thickness) (details in Supplementary Material).

The only increase in GM metrics in COVID-19 patients with respect to controls was found in the area of the right transverse temporal gyrus, with the highest values in the non-critical group (not correlated with inflammatory markers).

Critical COVID-19 patients had an increased burden of total $\mathrm{WMH}$, in particular periventricular WMH (Figure 3E). WMH volumes were positively correlated with inflammatory markers in the COVID-19 group (Total WMH with white cell count: $r$ $=0.36, p=0.012$; Total WMH with lymphocytes count: $r=$ $0.39, p=0.006$. PWMH with white cell count: $r=0.42, p=$ 0.003 ; neutrophils count: $r=0.32, p=0.025$; lymphocytes count: $r=0.42, p=0.003$; monocytes count: $r=0.30, p=0.036$ ) (Figure 3F).

\section{Diffusion-Weighted MR Imaging}

As previously shown by us, COVID-19 patients had increased mean diffusivity in the left posterior thalamic radiation and right sagittal stratum compared to controls (Figure 3G). When comparing severity groups, $\mathrm{MD}$ in the right sagittal stratum was higher in patients who received organ support with respect to both non-critical patients and controls (Figure $3 \mathbf{H}$ ). When looking at NAWM only, the results remained significant in the sagittal stratum (Figures 3J,K) and significantly higher MD was observed in the right posterior thalamic radiation in critical patients with respect to controls (Figures 3I,L). Higher MD was also observed in the NAWM of the left superior longitudinal fasciculus, positively correlated with monocyte count $(r=0.25$ $p=0.034)$.

\section{Susceptibility-Weighted MR Imaging}

COVID-19 patients had a higher $\mathrm{T}_{2}^{*}$ signal in the thalamus with respect to controls (left $p=0.052$; right $p=0.037$ ) (Figure $3 \mathbf{M}$ ), 


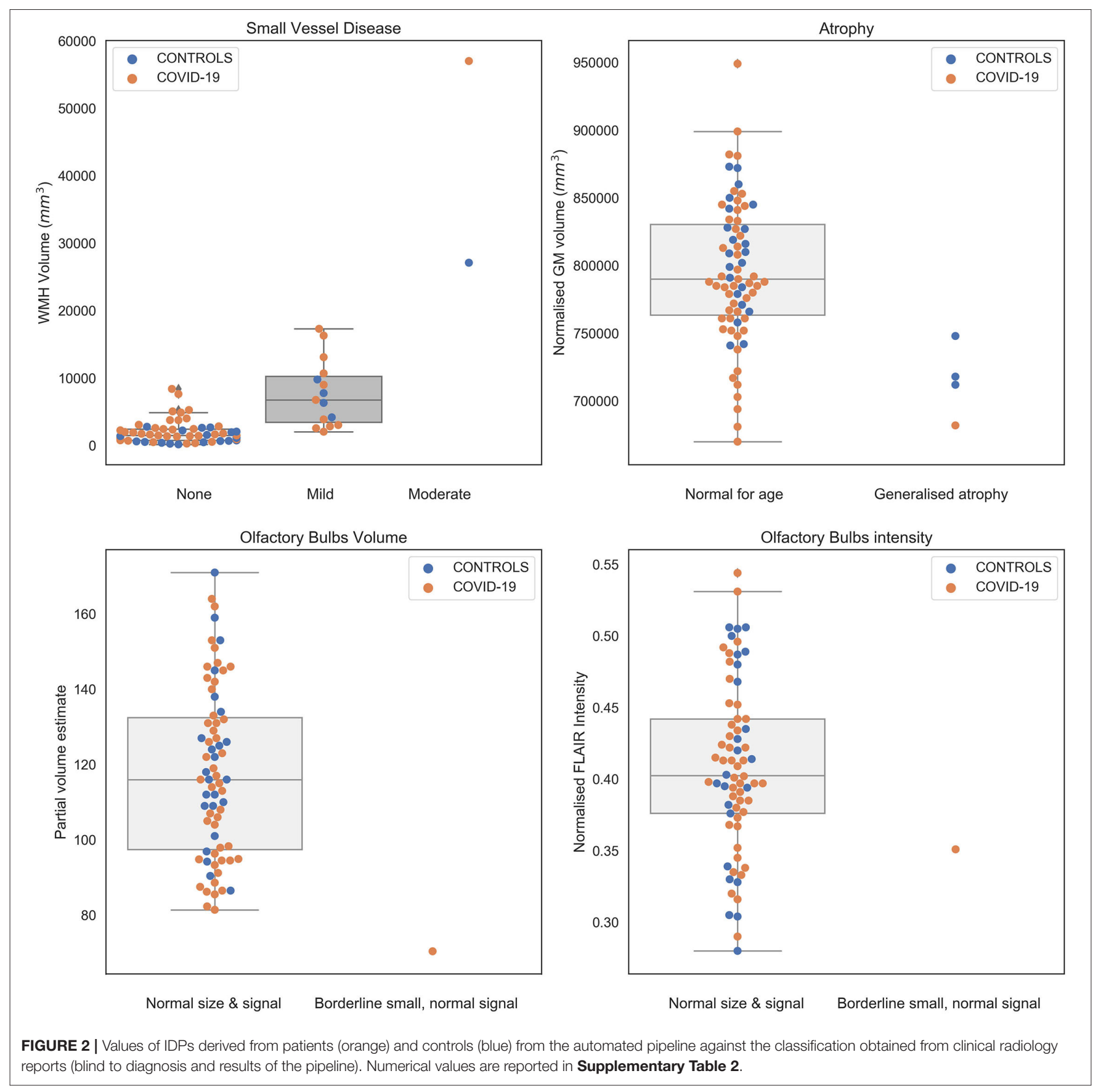

lower $\mathrm{T}_{2}^{*}$ in the left hippocampus. The comparison across severity groups revealed that the $\mathrm{T}_{2}^{*}$ signal in the right thalamus was higher in patients who did not receive organ support with respect to controls (Figure 3N). Within the COVID-19 patients, the $\mathrm{T}_{2}^{*}$ signal in the right thalamus was positively correlated with inflammation markers (white cell count: $r=0.30, p=0.036$; neutrophils count: $r=0.38, p=0.007$ ) (Figure 30).

\section{Arterial Spin Labelling}

No significant group differences were observed in CBF or ATT IDPs.

\section{Results of the Cohort Comparisons Based on Anosmia}

When looking at the olfactory system (olfactory bulbs and key GM areas) no significant differences were found across anosmia groups on the selected IDPs.

\section{DISCUSSION}

In this work we describe how we adapted the UKB brain MRI protocol and processing pipeline to multi-organ imaging of COVID-19, with initial direct application to the C-MORE study. 


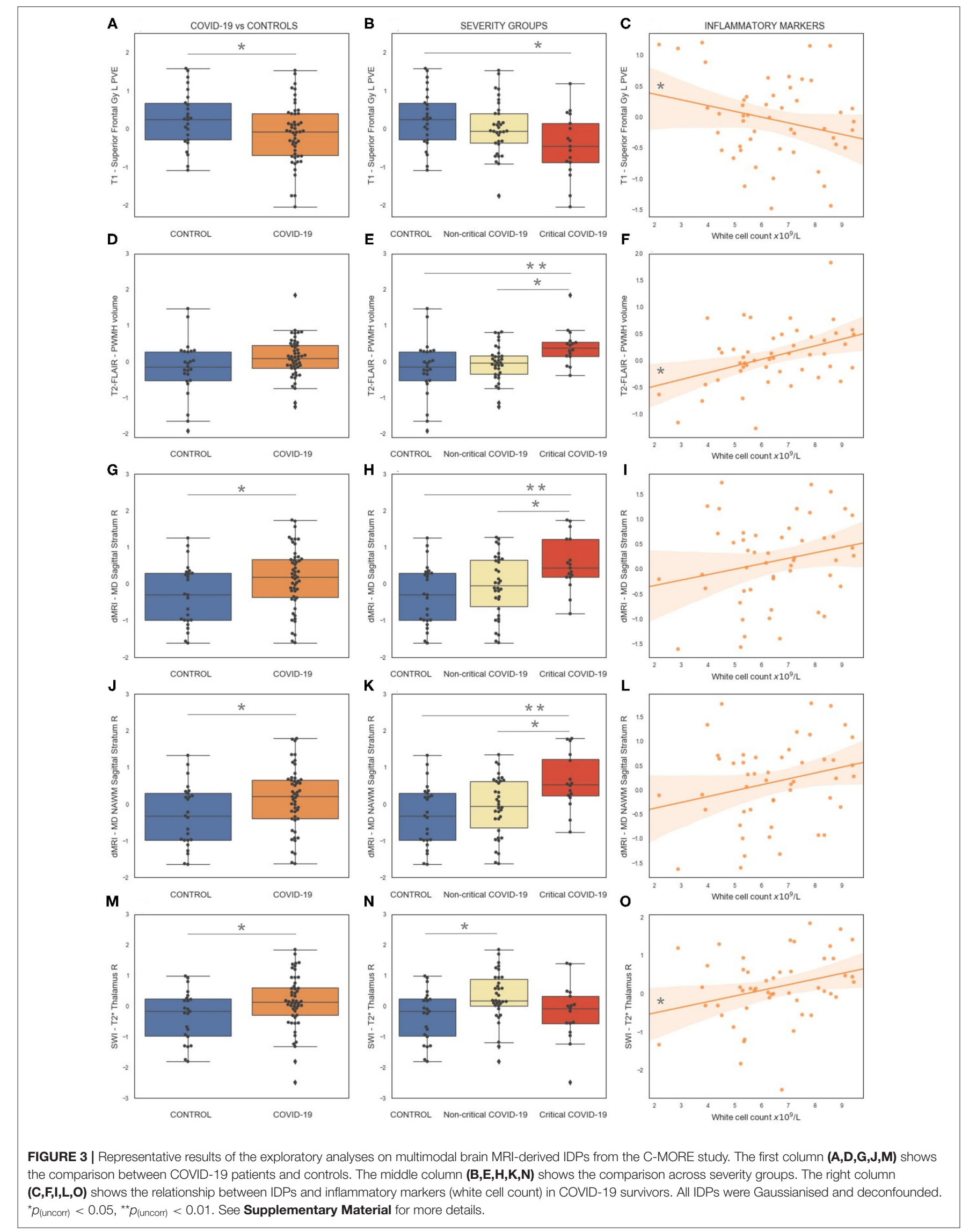


We developed a 17-min MRI protocol to study the effect of COVID-19 on the brain. By modifying the UK Biobank protocol, we were able to exploit its technical advances to generate high quality data in a reduced time, while tailoring it to the specific purpose of assessing the effects of COVID-19 (e.g., introducing ASL to assess brain perfusion). While some studies used multimodal MRI to look at differences between COVID19 patients and controls (6), to the best of our knowledge this is the most comprehensive brain imaging protocol adopted so far, including five MRI modalities. This will enable the monitoring of a wide range of potential medium and long-term effects of SARS-CoV-2 virus on the brain. This is also in line with the approach adopted by emerging consortia (61) which aim to develop neuroimaging common data elements that are capable of capturing the broad spectrum of brain imaging findings reported to date in adult patients with COVID-19 and that are feasible to implement in hospitals around the world, as already demonstrated in the UK-wide C-MORE study. In addition, the close similarity of our protocol to the UK Biobank protocol will enable utilisation of that dataset (e.g., for normative comodelling). We obtained usable data from $95 \%$ of participants, suggesting that the protocol is well-tolerated and can be used as part of a multiorgan MRI assessment.

The analysis pipeline currently generates 1,575 IDPs, including some specifically designed to look at potential effects of COVID-19. In particular, we were able to segment the olfactory bulbs, thanks to improvements in non-brain alignment and template construction, and we generated 8 IDPs from ASL. Many of these IDPs are also suitable for evaluating the feature-based common data elements proposed by GCS-NeuroCOVID for reporting MRI in COVID-19 (61).

For brain characteristics that are commonly reported by neuroradiologists, we compared our IDPs with clinical reports and found reasonable agreement, particularly for small vessel disease and atrophy. This suggests that the pipeline can be used to automatically extract meaningful information from larger datasets.

The first application of this protocol and pipeline was in the C-MORE study. Expanding the analysis performed in our recent work (3) by including new IDPs and exploring differences across disease groups and anosmia groups, we observed some interesting albeit preliminary group differences.

The differences we found in frontal GM (reduced in COVID19), WMHs (increased in COVID-19, especially periventricular $\mathrm{WMH}$ ) and MD (increased in COVID-19 in the sagittal stratum and posterior thalamic radiation, also evident when considering normal appearing WM only) were stronger in patients who received organ support, suggesting a relationship between disease severity and imaging biomarkers of neurological health. This is in line with a recent cohort study using electronic health records (9) showing a clear effect of COVID-19 severity on subsequent neurological diagnosis. Given the association of brain atrophy, vascular pathology, and WM microstructural damage on MRI with neurodegenerative diseases such as dementia, it would be prudent to monitor the long-term effects of such MRI abnormalities on cognitive performance of patients (11).
We found some brain IDPs to be correlated with inflammatory markers. A recent UK-wide follow up study of previously hospitalised COVID-19 patients $(N=1,077)$ have identified a subgroup of patients with cognitive impairment, increased markers of inflammation and high symptom burden (16). It is still currently unclear whether brain damage is caused by the viral infection itself (62) or by inflammatory reactions (63). The association between WMH and inflammatory markers like white cell count is likely to reflect a combination of hypercoagulable state acutely and chronic neuroinflammatory processes. Several studies have shown that the disease can cause inflammation and blood vessel damage and a recent post-mortem study found microvascular injury and inflammation but no signs of infection (64). The interpretation of the correlations with GM atrophy and $\mathrm{T}^{*}$ signal is more speculative. Since changes in vascularity can alter $\mathrm{T}^{*}$ signal, microvascular injury and inflammation could have altered $\mathrm{T}^{*}$ in the thalamus and a dysregulated inflammatory response in COVID-19 patients may be linked to changes in grey matter volume. More investigation is certainly needed and follow-up brain imaging will add considerable value to our understanding of the mechanisms underlying cognitive impairment in COVID-19 patients.

Neuropsychological and brain changes (including atrophy and WMHs) are known to occur in in patients admitted to ICU and survivors of critical illness $(65,66)$. Since we also observed differences in non-critical patients, our findings would suggest that these changes are not just a manifestation of post-ICU effects. Nevertheless, it can be argued that the controls in this study were not hospitalised and therefore it remains difficult to confidently attribute the observed differences to COVID19. It is also worth noting that the type of organ support that the 17 C-MORE COVID-19 patients received was quite heterogeneous (positive airway pressure ventilation, intubation, dialysis, vasopressor support, medical therapy), therefore further studies with larger samples may be required to disentangle effects of specific interventions.

Grey matter differences in non-frontal areas were instead more prominent in non-critical patients, suggesting a different pattern of the impact of COVID-19 on GM. Further investigation is needed to better characterise the cause and extent of this effect. The increased $\mathrm{T}_{2}^{*}$ in the thalamus was also higher in non-critical COVID-19 patients. Potential explanations are that more critical COVID-19 involved other brain changes and not the thalamus, or that the treatment (e.g., steroids) received by critical patients contributed to normalising $\mathrm{T}_{2}^{*}$ signal in this area. Pathological alterations in other deep structures are usually observed as reductions in $\mathrm{T}_{2}^{*}$ [e.g., due to iron accumulation (28)]. However, this opposite trend between the thalamus and other subcortical structures has been previously observed in UK Biobank data [see Figure 8 in (12)]: $\mathrm{T}_{2}^{*}$ in subcortical structures was found to be negatively correlated with age, while thalamus $\mathrm{T}_{2}^{*}$ showed a positive correlation with age. We also observed decreased $\mathrm{T}_{2}^{*}$ in the hippocampus in COVID-19 patients, which could be due to higher iron accumulation related to the virus infection. However, measurements in the hippocampus are likely to be affected by partial volume effect, due to the small size of the area and 
relatively thick slices. This is also an area that is prone to MRI signal dropout, so we cannot exclude this potential confound.

We specifically looked at the olfactory system (olfactory bulbs and key GM areas) and did not find differences between COVID-19 patients who experienced anosmia during their acute illness vs. those who did not and controls. Other studies have reported negative findings in people who experienced loss of smell $(6,20,21)$, though the majority are small in size and could have been underpowered. Future studies on larger samples are likely to be more sensitive to brain differences associated with anosmia or may find an absence of an anosmiaspecific radiological signature. As our analysis incorporated anosmia symptom data collected during admission and not at the time of the MRI, another possible explanation for a lack of differences could be that the symptoms (and associated radiological abnormalities) may have resolved by the time of the scan.

This exploratory study has several limitations. The sample size is relatively small and, due to the comparisons performed on a wide range of IDPs, the results do not survive correction for multiple comparison. Anosmia was not assessed at the time of scan but rather at admission. Group differences in brain IDPs between COVID subgroups could be related to the therapy used while in hospital and not specific to COVID-19 per se.

The C-MORE study included a limited number of previously hospitalised patients and focused on assessing medium-term damage using a univariate approach, but has paved the way for more extensive and longitudinal studies that can take advantage of the protocol and analysis developed as part of this study. The use of multivariate analysis approaches, especially on a bigger sample, might provide better sensitivity in associations with clinical variables. The follow-up phase of the C-MORE study is currently ongoing and aims to monitor the longitudinal trajectory of multiorgan health beyond the subacute phase. With more longitudinal clinical data available, multivariate analyses could also be a promising approach for predicting long-term outcome, e.g., risk of long-COVID. This multiorgan imaging protocol is also now being used in the "PHOSP-COVID Posthospitalisation COVID-19" study, a large UK-wide national consortium $(N=616)$ aiming to understand and improve long-term health outcomes for patients who have been in hospital with confirmed or suspected COVID-19, and in the CONVALESCENCE study, a study of patients with Long COVID $(N=800)$. This will allow for testing the reproducibility of our preliminary results and will augment our understanding of the medium to long term neurological sequelae of COVID-19.

Finally, UK Biobank is imaging 3,000 volunteers from the 45,000 subjects that were already scanned before the pandemic. Half of these will have been invited for scanning because tests show that they have been exposed to the virus and became infected (while the other half will be a control group). This pre- vs. post-COVID-19 imaging data will be a large and rich resource for studying the effects of this disease in the brain and other parts of the body, also expanding our knowledge of the impact of COVID-19 on people who were not hospitalised. The preprocessing and analyses of these data is already benefiting from the work described above (67).
In this work we presented the rationale behind the choices made for this protocol at the beginning of the study. In light of the results obtained here and more recently in the UKB prevs-post COVID study (67), some considerations can be made to inform future research. First, $\mathrm{T}_{1}$ and $\mathrm{T}_{2}$-FLAIR sequences proved to be very valuable to detect atrophy and markers of brain inflammation in COVID-19 patients. Second, diffusion-weighted MRI is important for looking at microstructural tissue damage (and potentially inflammation, as well as changes in diffusion metrics due to changes in tissue volume and partial voluming). In this study we used only 3 directions to reduce acquisition time, but adding a higher quality dMRI sequence (e.g., like the 7-min one used in UKB) could benefit future studies, albeit at the cost of slightly longer scan time. Finally, having multiple imaging time points after infection would be valuable, even without preCOVID imaging, as biological effects are likely to change over time after infection.

In conclusion, we developed a brain MRI protocol and analysis pipeline that enable efficient yet comprehensive assessment of brain characteristics in COVID-19 patients, and can be used both as part of a multiorgan imaging study as well as standalone. The availability of the protocol, code, and data will further contribute to the understanding of the medium to long-term effects of COVID-19.

\section{DATA AVAILABILITY STATEMENT}

The datasets presented in this study can be found in online repositories. The names of the repository/repositories and accession number(s) can be found at: Gitlab (https:/git. fmrib.ox.ac.uk/open-science/analysis/c-more-brain-mri); OraData (https://doi.org/10.5287/bodleian:prqXzwj7N).

\section{ETHICS STATEMENT}

The studies involving human participants were reviewed and approved by the North West Preston Research Ethics Committee (reference 20/NW/0235). This study was also registered at ClinicalTrials.gov (NCT04510025). The patients/participants provided their written informed consent to participate in this study.

\section{AUTHOR CONTRIBUTIONS}

LG: conceptualisation, methodology, software, investigation, data curation, formal analysis, visualisation, and writingoriginal draft. BR: conceptualisation, investigation, data curation, project administration, funding acquisition, and writing-review and editing. NF: investigation, data curation, formal analysis, visualisation, and writing-review and editing. FA-A: software, data curation, formal analysis, and writingreview and editing. MPC: conceptualisation, investigation, data curation, project administration, and writing-review and editing. FS and MR: methodology, investigation, and writing-review and editing. TO, FK, MAC, CW, CA, and FL: software, investigation, and writing-review and editing. 
JA and ET: methodology, software, and writing-review and editing. CM: resources, funding acquisition, and writing-review and editing. SN: conceptualisation, methodology, supervision, funding acquisition, and writing-review and editing. KM: conceptualisation, methodology, investigation, and writingreview and editing. PJ: conceptualisation, methodology, and writing-review and editing. SS: conceptualisation, methodology, investigation, resources, supervision, funding acquisition, and writing-review and editing. All authors contributed to the article and approved the submitted version.

\section{FUNDING}

This work was supported by NIHR Oxford and Oxford Health Biomedical Research Centre, Oxford British Heart Foundation (BHF) Centre of Research Excellence (RE/18/3/34214), United Kingdom Research Innovation, Wellcome Trust, and British Heart Foundation. This project was part of a tier 3 study (C-MORE) within the collaborative research programme entitled PHOSP-COVID Post-hospitalisation COVID-19 study: a national consortium to understand and improve long-term health outcomes. Funded by the Medical Research Council and Department of Health and Social Care/National Institute for Health Research Grant (MR/V027859/1) ISRCTN number 10980107. This work also arises from one of the national COVID-19 Cardiovascular Disease Flagship Projects designated by the NIHR-BHF Cardiovascular Partnership. PJ thanks the Dunhill Medical Trust for support. MAC and FK acknowledge support from EPSRC (EP/P012361/1). TO was supported by a Sir Henry Dale Fellowship jointly funded by the Wellcome Trust and the Royal Society (220204/Z/20/Z). The Wellcome Centre for Integrative Neuroimaging was supported by core funding from the Wellcome Trust (203139/Z/16/Z). The authors

\section{REFERENCES}

1. Huang C, Wang Y, Li X, Ren L, Zhao J, Hu Y, et al. Clinical features of patients infected with 2019 novel coronavirus in Wuhan, China. Lancet. (2020) 395:497-506. doi: 10.1016/S0140-6736(20)30183-5

2. Guan WJ, Ni ZY, Hu Y, Liang WH, Ou CQ, He JX, et al. Clinical characteristics of coronavirus disease 2019 in China. N Engl J Med. (2020) 382:170820. doi: 10.1056/NEJMoa2002032

3. Raman B, Cassar MP, Tunnicliffe EM, Filippini N, Griffanti L, AlfaroAlmagro F, et al. Medium-term effects of SARS-CoV-2 infection on multiple vital organs, exercise capacity, cognition, quality of life and mental health, post-hospital discharge. EClinicalMedicine. (2021) 31:100683. doi: 10.1016/j.eclinm.2020.100683

4. Choi Y, Lee MK. Neuroimaging findings of brain MRI and CT in patients with COVID-19: a systematic review and meta-analysis. Eur J Radiol. (2020) 133:109393. doi: 10.1016/j.ejrad.2020.10 9393

5. Sawlani V, Scotton S, Nader K, Jen JP, Patel M, Gokani K, et al. COVID-19-related intracranial imaging findings: a large single-centre experience. Clin Radiol. (2021) 76:108-16. doi: 10.1016/j.crad.2020.0 9.002

6. Lu Y, Li X, Geng D, Mei N, Wu PY, Huang CC, et al. Cerebral microstructural changes in COVID-19 patients - an MRI-based 3-month followup study. EClinicalMedicine. (2020) 25:100484. doi: 10.1016/j.eclinm.2020.10 0484 gratefully acknowledge funding from the Wellcome Trust Collaborative Award (215573/Z/19/Z). KM acknowledges further support from the Wellcome Trust Senior Research Fellowship (202788/Z/16/Z). This research was funded in part by the Wellcome Trust (Grants numbers 203139/Z/16/Z, $215573 / Z / 19 / Z, 220204 / Z / 20 / Z$, and $202788 / Z / 16 / Z)$. For the purpose of open access, the authors have applied a CC BY public copyright licence to any author accepted manuscript version arising from this submission.

\section{ACKNOWLEDGMENTS}

We thank our patients and their families who have sought to help others understand the effects of COVID-19. We are grateful to the University of Oxford and Oxford University Hospital Trust for their support of this study. We would like to acknowledge OCMR staff, Ms. Rebecca Mills, Ms. Polly Whitworth, Ms. Joana Leal Pelado, Ms. Claudia Nunes, Ms. Harriet Nixon, Ms. Injung Jang, Ms. Maryam Khan, Ms. Gillian Roberts, Mr. Mike MacDonald, Ms. Jasmine Taylor, Dr. Ahmad Moolla, Ms. Sarah White, and Dr. Ines Abdesselam, for their help with this work. We thank Hanan Lamlum for her help in application of ethics. We acknowledge the support of Siemens in providing a licence for the use of ASL. The diffusion sequence used in our protocol was kindly provided by the CMRR, University of Minnesota, USA.

\section{SUPPLEMENTARY MATERIAL}

The Supplementary Material for this article can be found online at: https://www.frontiersin.org/articles/10.3389/fneur. 2021.753284/full\#supplementary-material

7. Ellul MA, Benjamin L, Singh B, Lant S, Michael BD, Easton A, et al. Neurological associations of COVID-19. Lancet Neurol. (2020) 19:76783. doi: 10.1016/S1474-4422(20)30221-0

8. Varatharaj A, Thomas N, Ellul MA, Davies NWS, Pollak TA, Tenorio EL, et al. Neurological and neuropsychiatric complications of COVID-19 in 153 patients: a UK-wide surveillance study. Lancet Psychiatry. (2020) 7:87582. doi: 10.1016/S2215-0366(20)30287-X

9. Taquet M, Geddes JR, Husain M, Luciano S, Harrison PJ. 6-month neurological and psychiatric outcomes in 236379 survivors of COVID-19: a retrospective cohort study using electronic health records. Lancet Psychiatry. (2021) 8:416-27. doi: 10.1016/S2215-0366(21)00084-5

10. Mao L, Jin $\mathrm{H}$, Wang $\mathrm{M}, \mathrm{Hu} \mathrm{Y}$, Chen $\mathrm{S}, \mathrm{He} \mathrm{Q}$, et al. Neurologic manifestations of hospitalized patients with coronavirus disease 2019 in Wuhan, China. JAMA Neurol. (2020) 77:683-90. doi: 10.1001/jamaneurol.20 20.1127

11. Miners S, Kehoe PG, Love S. Cognitive impact of COVID19: looking beyond the short term. Alzheimers Res Ther. (2020) 12:170. doi: 10.1186/s13195-020-00744-w

12. Miller KL, Alfaro-Almagro F, Bangerter NK, Thomas DL, Yacoub E, Xu J, et al. Multimodal population brain imaging in the UK Biobank prospective epidemiological study. Nat Neurosci. (2016) 19:1523-36. doi: 10.1038/nn.4393

13. Alfaro-Almagro F, Jenkinson M, Bangerter NK, Andersson JLR, Griffanti L, Douaud G, et al. Image processing and quality control for the first 10,000 brain imaging datasets from UK Biobank. NeuroImage. (2018) 166:40024. doi: 10.1016/j.neuroimage.2017.10.034 
14. Pini L, Pievani M, Bocchetta M, Altomare D, Bosco P, Cavedo E, et al. Brain atrophy in alzheimer's disease and aging. Ageing Res Rev. (2016) 30:2548. doi: 10.1016/j.arr.2016.01.002

15. Kremer S, Lersy F, de Seze J, Ferre JC, Maamar A, Carsin-Nicol B, et al. Brain MRI findings in severe COVID-19: a retrospective observational study. Radiology. (2020) 297:E242-51. doi: 10.1148/radiol.2020202222

16. PHOSP-COVID Collaborative Group, Evans RA, McAuley H, Harrison EM, Shikotra A, Singapuri A, et al. Physical, cognitive and mental health impacts of COVID-19 following hospitalisation - a multi-centre prospective cohort study. medRxiv. (2021). doi: 10.1101/2021.03.22.21254057

17. Egbert AR, Cankurtaran S, Karpiak S. Brain abnormalities in COVID-19 acute/subacute phase: a rapid systematic review. Brain Behav Immun. (2020) 89:543-54. doi: 10.1016/j.bbi.2020.07.014

18. Lin E, Lantos JE, Strauss SB, Phillips CD, Campion TR Jr, Navi BB, et al. Brain imaging of patients with COVID-19: findings at an academic institution during the height of the outbreak in New York City. AJNR Am J Neuroradiol. (2020) 41:2001-8. doi: 10.3174/ajnr.A6793

19. Chiu A, Fischbein N, Wintermark M, Zaharchuk G, Yun PT, Zeineh M. COVID-19-induced anosmia associated with olfactory bulb atrophy. Neuroradiology. (2021) 63:147-8. doi: 10.1007/s00234-020-02554-1

20. Galougahi MK, Ghorbani J, Bakhshayeshkaram M, Naeini AS, Haseli S. Olfactory bulb magnetic resonance imaging in SARSCoV-2-induced anosmia: the first report. Acad Radiol. (2020) 27:892-3. doi: 10.1016/j.acra.2020.04.002

21. Shor N, Chougar L, Pyatigorskaya N. MR imaging of the olfactory bulbs in patients with COVID-19 and anosmia: how to avoid misinterpretation. AJNR Am J Neuroradiol. (2021) 42:E10-1. doi: 10.3174/ajnr.A6921

22. Beauchamp LC, Finkelstein DI, Bush AI, Evans AH, Barnham KJ. Parkinsonism as a third wave of the COVID-19 pandemic? J Parkinsons Dis. (2020) 10:1343-53. doi: 10.3233/JPD-202211

23. Sulzer D, Antonini A, Leta V, Nordvig A, Smeyne RJ, Goldman JE, et al. COVID-19 and possible links with Parkinson's disease and parkinsonism: from bench to bedside. NPJ Parkinsons Dis. (2020) 6:18. doi: 10.1038/s41531-020-00123-0

24. Mittal S, Wu Z, Neelavalli J, Haacke EM. Susceptibility-weighted imaging: technical aspects and clinical applications, part 2. AJNR Am J Neuroradiol. (2009) 30:232-52. doi: 10.3174/ajnr.A1461

25. Wang C, Foxley S, Ansorge O, Bangerter-Christensen S, Chiew M, Leonte A, et al. Methods for quantitative susceptibility and R2* mapping in whole postmortem brains at 7T applied to amyotrophic lateral sclerosis. NeuroImage. (2020) 222:117216. doi: 10.1016/j.neuroimage.2020.117216

26. Conklin J, Frosch MP, Mukerji SS, Rapalino O, Maher MD, Schaefer PW, et al. Susceptibility-weighted imaging reveals cerebral microvascular injury in severe COVID-19. J Neurol Sci. (2021) 421:117308. doi: 10.1016/j.jns.2021.117308

27. Harder SL, Hopp KM, Ward H, Neglio H, Gitlin J, Kido D. Mineralization of the deep gray matter with age: a retrospective review with susceptibility-weighted MR imaging. AJNR Am J Neuroradiol. (2008) 29:176-83. doi: 10.3174/ajnr.A0770

28. Ward RJ, Zucca FA, Duyn JH, Crichton RR, Zecca L. The role of iron in brain ageing and neurodegenerative disorders. Lancet Neurol. (2014) 13:104560. doi: 10.1016/S1474-4422(14)70117-6

29. Okell TW, Chappell MA, Kelly ME, Jezzard P. Cerebral blood flow quantification using vessel-encoded arterial spin labeling. J Cereb Blood Flow Metab. (2013) 33:1716-24. doi: 10.1038/jcbfm.2013.129

30. Jenkinson M, Beckmann CF, Behrens TE, Woolrich MW, Smith SM. FSL. NeuroImage. (2012) 62:782-90. doi: 10.1016/j.neuroimage.2011.09.015

31. Jenkinson $M$, Smith S. A global optimisation method for robust affine registration of brain images. Med Image Anal. (2001) 5:143-56. doi: 10.1016/S1361-8415(01)00036-6

32. Andersson JLR, Jenkinson M, Smith S. High resolution nonlinear registration with simultaneous modelling of intensities. bioRxiv. (2019). doi: 10.1101/646802

33. Lange FJ, Ashburner J, Smith SM, Andersson JLR. A symmetric prior for the regularisation of elastic deformations: improved anatomical plausibility in nonlinear image registration. NeuroImage. (2020) 219:116962. doi: 10.1016/j.neuroimage.2020.116962
34. Smith SM, Zhang Y, Jenkinson M, Chen J, Matthews PM, Federico A, et al. Accurate, robust, and automated longitudinal and cross-sectional brain change analysis. NeuroImage. (2002) 17:479-89. doi: 10.1006/nimg.2002.1040

35. Zhang Y, Brady M, Smith S. Segmentation of brain MR images through a hidden Markov random field model and the expectation-maximization algorithm. IEEE Trans Med Imaging. (2001) 20:45-57. doi: 10.1109/42.906424

36. Patenaude B, Smith SM, Kennedy DN, Jenkinson M. A Bayesian model of shape and appearance for subcortical brain segmentation. NeuroImage. (2011) 56:907-22. doi: 10.1016/j.neuroimage.2011.02.046

37. Fischl B, van der Kouwe A, Destrieux C, Halgren E, Segonne F, Salat DH, et al. Automatically parcellating the human cerebral cortex. Cereb Cortex. (2004) 14:11-22. doi: 10.1093/cercor/bhg087

38. Desikan RS, Segonne F, Fischl B, Quinn BT, Dickerson BC, Blacker D, et al. An automated labeling system for subdividing the human cerebral cortex on MRI scans into gyral based regions of interest. NeuroImage. (2006) 31:96880. doi: 10.1016/j.neuroimage.2006.01.021

39. Fischl B, Salat DH, Busa E, Albert M, Dieterich M, Haselgrove $\mathrm{C}$, et al. Whole brain segmentation: automated labeling of neuroanatomical structures in the human brain. Neuron. (2002) 33:341-55. doi: 10.1016/S0896-6273(02)00569-X

40. Iglesias JE, Augustinack JC, Nguyen K, Player CM, Player A, Wright M, et al. A computational atlas of the hippocampal formation using ex vivo, ultrahigh resolution MRI: application to adaptive segmentation of in vivo MRI. NeuroImage. (2015) 115:117-37. doi: 10.1016/j.neuroimage.2015.04.042

41. Klein A, Tourville J. 101 labeled brain images and a consistent human cortical labeling protocol. Front Neurosci. (2012) 6:171. doi: 10.3389/fnins.2012.00171

42. Smith SM, Alfaro-Almagro F, Miller KL. UK Biobank Brain Imaging Documentation - Version 1.8 (2020). Available online at: https://biobank.ctsu. ox.ac.uk/crystal/crystal/docs/brain_mri.pdf

43. Griffanti L, Zamboni G, Khan A, Li L, Bonifacio G, Sundaresan V, et al. BIANCA (Brain Intensity AbNormality Classification Algorithm): a new tool for automated segmentation of white matter hyperintensities. NeuroImage. (2016) 141:191-205. doi: 10.1016/j.neuroimage.2016.07.018

44. Griffanti L, Jenkinson M, Suri S, Zsoldos E, Mahmood A, Filippini N, et al. Classification and characterization of periventricular and deep white matter hyperintensities on MRI: a study in older adults. NeuroImage. (2018) 170:17481. doi: 10.1016/j.neuroimage.2017.03.024

45. DeCarli C, Fletcher E, Ramey V, Harvey D, Jagust WJ. Anatomical mapping of white matter hyperintensities (WMH): exploring the relationships between periventricular WMH, deep WMH, and total WMH burden. Stroke. (2005) 36:50-5. doi: 10.1161/01.STR.0000150668.58689.f2

46. Fonov V, Evans AC, Botteron K, Almli CR, McKinstry RC, Collins DL, et al. Unbiased average age-appropriate atlases for pediatric studies. NeuroImage. (2011) 54:313-27. doi: 10.1016/j.neuroimage.2010.07.033

47. Arthofer C, Smith SM, Jenkinson M, Andersson J, Lange F, editors. Multimodal MRI Template Construction From UK Biobank: Oxford-MM-O. Organisation for Human Brain Mapping (OHBM) (2021).

48. Grabner G, Janke AL, Budge MM, Smith D, Pruessner J, Collins DL. Symmetric atlasing and model based segmentation: an application to the hippocampus in older adults. Med Image Comput Comput Assist Interv. (2006) 9(Pt 2):58-66. doi: 10.1007/11866763_8

49. Jenkinson M, Bannister P, Brady M, Smith S. Improved optimization for the robust and accurate linear registration and motion correction of brain images. NeuroImage. (2002) 17:825-41. doi: 10.1006/nimg.2002.1132

50. Andersson JL, Skare S, Ashburner J. How to correct susceptibility distortions in spin-echo echo-planar images: application to diffusion tensor imaging. NeuroImage. (2003) 20:870-88. doi: 10.1016/S1053-8119(03)00 336-7

51. Wang CS, Smith SM, Alfaro-Almagro F, Fiscone C, Bowtell R, Elliott L, et al. editor. Quantitative susceptibility mapping in UK Biobank brain imaging: pipeline and preliminary results in 2400 subjects. In: ISMRM (2020).

52. Eckstein K, Dymerska B, Bachrata B, Bogner W, Poljanc K, Trattnig S, et al. Computationally efficient combination of multi-channel phase data from multi-echo acquisitions (ASPIRE). Mag Reson Med. (2018) 79:29963006. doi: 10.1002/mrm.26963

53. Schofield MA, Zhu Y. Fast phase unwrapping algorithm for interferometric applications. Opt Lett. (2003) 28:1194-6. doi: 10.1364/OL.28.001194 
54. Wu B, Li W, Avram AV, Gho SM, Liu C. Fast and tissue-optimized mapping of magnetic susceptibility and $\mathrm{T} 2 *$ with multi-echo and multi-shot spirals. NeuroImage. (2012) 59:297-305. doi: 10.1016/j.neuroimage.2011.07.019

55. Schweser F, Deistung A, Lehr BW, Reichenbach JR. Quantitative imaging of intrinsic magnetic tissue properties using MRI signal phase: an approach to in vivo brain iron metabolism? NeuroImage. (2011) 54:2789807. doi: 10.1016/j.neuroimage.2010.10.070

56. Li W, Wang N, Yu F, Han H, Cao W, Romero R, et al. A method for estimating and removing streaking artifacts in quantitative susceptibility mapping. NeuroImage. (2015) 108:111-22. doi: 10.1016/j.neuroimage.2014.12.043

57. Llera A, Vidaurre D, Pruim R, Beckmann C. Variational mixture models with gamma or inverse-gamma components. arXiv preprint. (2016) arXiv:160707573.

58. Chappell MA, Groves AR, Whitcher B, Woolrich MW. Variational Bayesian inference for a nonlinear forward model. IEEE Trans Signal Process. (2008) 57:223-36. doi: 10.1109/TSP.2008.2005752

59. Chappell MA, MacIntosh BJ, Donahue MJ, Gunther M, Jezzard P, Woolrich MW. Separation of macrovascular signal in multi-inversion time arterial spin labelling MRI. Mag Reson Med. (2010) 63:1357-65. doi: 10.1002/mrm.22320

60. Chappell MA, Groves AR, MacIntosh BJ, Donahue MJ, Jezzard P, Woolrich MW. Partial volume correction of multiple inversion time arterial spin labeling MRI data. Mag Reson Med. (2011) 65:117383. doi: $10.1002 / \mathrm{mrm} .22641$

61. Edlow BL, Boly M, Chou SH, Fischer D, Kondziella D, Li LM, et al. Common data elements for COVID-19 neuroimaging: a GCS-NeuroCOVID proposal. Neurocrit Care. (2021) 34:365-70. doi: 10.1007/s12028-021-01192-6

62. de Erausquin GA, Snyder H, Carrillo M, Hosseini AA, Brugha TS, Seshadri $\mathrm{S}$, et al. The chronic neuropsychiatric sequelae of COVID-19: the need for a prospective study of viral impact on brain functioning. Alzheimers Dement. (2021) 17:1056-65. doi: 10.1002/alz.12255

63. Yang AC, Kern F, Losada PM, Agam MR, Maat CA, Schmartz GP, et al. Dysregulation of brain and choroid plexus cell types in severe COVID-19. Nature. (2021) 595:565-71. doi: 10.1038/s41586-021-03710-0

64. Lee MH, Perl DP, Nair G, Li W, Maric D, Murray H, et al. Microvascular injury in the brains of patients with Covid-19. N Engl J Med. (2021) 384:4813. doi: 10.1056/NEJMc2033369

65. Jackson JC, Pandharipande PP, Girard TD, Brummel NE, Thompson JL, Hughes CG, et al. Depression, post-traumatic stress disorder, and functional disability in survivors of critical illness in the BRAIN-ICU study: a longitudinal cohort study. Lancet Respir Med. (2014) 2:36979. doi: 10.1016/S2213-2600(14)70051-7

66. Suchyta MR, Jephson A, Hopkins RO. Neurologic changes during critical illness: brain imaging findings and neurobehavioral outcomes. Brain Imaging Behav. (2010) 4:22-34. doi: 10.1007/s11682-009-9082-3

67. Douaud G, Lee S, Alfaro-Almagro F, Arthofer C, Wang C, Lange F, et al. Brain imaging before and after COVID-19 in UK Biobank. medRxiv. (2021). doi: 10.1101/2021.06.11.21258690
Conflict of Interest: BR reports grants from NIHR Oxford Biomedical Research Centre, grants from United Kingdom Research Innovation Award, and Oxford British Heart Foundation Centre for Research Excellence during the conduct of the study. MPC reports grants from NIHR Oxford Biomedical Research Centre, during the conduct of the study. ET reports grants from NIHR Oxford Biomedical Research Centre, during the conduct of the study; shareholding in Perspectum, outside the submitted work. TO reports grants from Wellcome Trust/Royal Society, during the conduct of the study; personal fees from SBGNeuro, personal fees from Oxford University Press, personal fees from Siemens Healthineers, outside the submitted work; In addition, outside the submitted work, TO has a patent Combined angiography and perfusion using radial imaging and arterial spin labelling pending, a patent (with PJ) Off-resonance Correction for Pseudocontinuous Arterial Spin Labelling pending, a patent Estimation of blood flow rates issued, a patent (with MAC) Fast analysis method for non-invasive imaging of blood flow using vessel-encoded arterial spin labelling with royalties paid by Siemens Healthineers, and a patent (with PJ and MAC) Quantification of blood volume flow rates from dynamic angiography data with royalties paid by Siemens Healthineers. MAC reports personal fees from Oxford University Press and Springer-Nature, outside submitted work. SN reports grants from Oxford NIHR Biomedical Research Centre, grants from UKRI, during the conduct of the study; personal fees, and others from Perspectum Diagnostics, outside the submitted work. PJ reports grants from Oxford NIHR Biomedical Research Centre and salary support from the Dunhill Medical Trust. LG, FA-A, MAC, $\mathrm{JA}$, and SS receive royalties from licencing of FSL to non-academic, commercial entities.

The remaining authors declare that the research was conducted in the absence of any commercial or financial relationships that could be construed as a potential conflict of interest.

Publisher's Note: All claims expressed in this article are solely those of the authors and do not necessarily represent those of their affiliated organizations, or those of the publisher, the editors and the reviewers. Any product that may be evaluated in this article, or claim that may be made by its manufacturer, is not guaranteed or endorsed by the publisher.

Copyright (c) 2021 Griffanti, Raman, Alfaro-Almagro, Filippini, Cassar, Sheerin, Okell, Kennedy McConnell, Chappell, Wang, Arthofer, Lange, Andersson, Mackay, Tunnicliffe, Rowland, Neubauer, Miller, Jezzard and Smith. This is an open-access article distributed under the terms of the Creative Commons Attribution License (CC $B Y)$. The use, distribution or reproduction in other forums is permitted, provided the original author(s) and the copyright owner(s) are credited and that the original publication in this journal is cited, in accordance with accepted academic practice. No use, distribution or reproduction is permitted which does not comply with these terms. 